\title{
Combination pretest probability assessment and D-dimer did not reduce outpatient imaging for venous thromboembolism in a tertiary care hospital emergency department
}

\author{
Sarah Ingber, MD*; Rita Selby, MD, MSc ${ }^{\dagger \ddagger}$; Jacques Lee, MD, MSc ${ }^{\S}$; William Geerts, $\mathrm{MD}^{\dagger}$; \\ Elena Brnjac, $\mathrm{MLT}^{\ddagger}$
}

\section{ABSTRACT}

Introduction: Venous thromboembolism (VTE) is difficult to diagnose yet potentially life threatening. A low-risk pretest probability (PTP) assessment combined with a negative Ddimer can rule out VTE in two-thirds of outpatients, reducing the need for imaging. Real-life implementation of this strategy is associated with several challenges.

Methods: We evaluated the impact of introducing a standardized diagnostic algorithm including a mandatory PTP assessment and D-dimer on radiologic test use for VTE in our emergency department (ED). A retrospective review of all ED visits for suspected VTE in the year prior to and following the introduction of this algorithm was conducted. VTE diagnosis was based on imaging. Guideline compliance was also assessed.

Results: ED visits were investigated for suspected VTE in the pre- and postintervention periods $(n=1,785)$. Most D-dimers $(95 \%)$ ordered were associated with a PTP assessment, and $50 \%$ of visits assigned a low PTP had a negative D-dimer. The proportion of imaging tests ordered for VTE in all ED visits was unchanged postintervention (1.9\% v. $2.0 \%$ ). The proportion of patients with suspected VTE in whom VTE was confirmed on imaging decreased postintervention (10.2\% v. 14.1\%).

Conclusion: In spite of excellent compliance with our algorithm, we were unable to reduce imaging for VTE. This may be due to a lower threshold for suspecting VTE and an increase in investigation for VTE combined with a high false positive rate of our D-dimer assay in low-pretest probability patients. This study highlights two common real-life challenges with adopting this strategy for VTE investigation.

\section{RÉSUMÉ}

Introduction: Les thromboembolies veineuses (TEV) sont difficiles à diagnostiquer, pourtant elles peuvent être mortelles. Une évaluation du risque avant intervention (ERAI) considérée comme faible, associée à l'absence de Ddimères permet d'écarter la présence d'une TEV chez les deux tiers des malades externes, d'où diminution de la nécessité de recourir aux examens par imagerie. Toutefois, I'application de cette nouvelle façon de faire soulève, en pratique, plusieurs problèmes.

Méthodes: II y a eu détermination de l'incidence de I'application d'un nouvel algorithme de diagnostic uniformisé, comportant une ERAl obligatoire et un dosage des Ddimères, sur le recours aux examens radiologiques, dans les cas de TEV, au service des urgences (SU) de notre établissement. L'étude consistait en un examen rétrospectif de toutes les consultations faites au SU, dans les cas présumés de TEV, au cours de l'année précédant et suivant l'application de ce nouvel algorithme. Le diagnostic de TEV reposait sur l'imagerie. A aussi été évalué le respect des lignes directrices.

Résultats: Les consultations au SU pour des TEV présumées au cours des périodes précédant et suivant l'intervention ( $n$ $=1,785$ ) ont fait l'objet d'examen. La plupart des demandes de dosage des D-dimères (95\%) faisaient suite à I'ERAl, et $50 \%$ des patients considérés comme à faible risque ont obtenu des résultats négatifs à la recherche des $\mathrm{D}$-dimères. La proportion des demandes d'examen par imagerie pour une TEV par rapport à toutes les consultations au SU n'a pas changé au cours de la période après intervention $11.9 \%$ contre [c.] 2.0\%), mais la proportion de patients souffrant d'une TEV présumée chez qui l'accident thrombotique a été confirmé à l'imagerie a diminué au cours de la période après intervention ( $10.2 \%$ c. $14.1 \%)$.

Conclusion: Malgré le respect rigoureux de l'algorithme, il a été impossible de diminuer le nombre de demandes d'examen par imagerie dans les cas de TEV. Cela peut

From the *Division of Hematology, University of Toronto, and Departments of †Medicine $\ddagger$ Clinical Pathology, and §Emergency Medicine, Sunnybrook Health Sciences Centre, University of Toronto, Toronto, ON.

Correspondence to: Dr. Rita Selby, Coagulation Laboratories, Sunnybrook Health Sciences Centre and University Health Network, D-675a, 2075 Bayview Avenue, Toronto, ON M4N 3M5; rita.selby@sunnybrook.ca.

This article has been peer reviewed. 
s'expliquer par une diminution du nombre de cas présumés de TEV et par une augmentation du nombre de cas soumis à des examens exploratoires en vue de confirmer ou d'infirmer une TEV, associées à un taux élevé de faux positifs du dosage des $D$-dimères chez les patients ayant un faible risque de TEV avant intervention. L'étude fait ressortir, en pratique, deux difficultés courantes de l'application de cette nouvelle démarche dans l'exploration des TEV.

Keywords: D-dimer, quality improvement, venous thromboembolism
Making the diagnosis of deep vein thrombosis (DVT) and pulmonary embolism (PE) can be challenging in the emergency department (ED). Imaging tests used to rule out these diagnoses include duplex ultrasonography (DUS), computed tomographic pulmonary angiography (CTPA), and ventilation perfusion ( $(\dot{\mathrm{V}} / \dot{\mathrm{Q}})$ scans. These tests are inconvenient and expensive, and CTPA increases patient exposure to radiation. Moreover, their diagnostic yield is only 5 to $25 \%$, indicating that many patients spend prolonged time in the ED and are exposed to risks unnecessarily. ${ }^{1}$

To reduce the use of low-yield imaging studies to rule out venous thromboembolism (VTE), diagnostic strategies that combine the use of standardized clinical pretest probability (PTP) assessments with D-dimer testing have been developed. ${ }^{2-4}$ Studies have shown that low clinical pretest probability for VTE combined with a negative, highly sensitive $\mathrm{D}$-dimer assay can safely rule out VTE in low-risk patients. ${ }^{5,6}$ The rate of thromboembolic events in this population when anticoagulation is withheld on this basis is low $(0.45 \%)$, and this strategy has been found to be cost effective. ${ }^{7,8}$ Real-life studies have identified challenges in implementing this diagnostic strategy, including inappropriate use of D-dimer testing in high-risk patients, noncompliance with the algorithms, difficulties with validating a cutoff value for $\mathrm{D}$-dimer tests, false positive D-dimer tests, use of inappropriate lowsensitivity D-dimer assays, and a reduction in the threshold for investigating VTE leading to an increase in the use of diagnostic imaging. ${ }^{9-12}$

The objective of our study was to assess whether the introduction of a standardized clinical PTP assessment prior to ordering of D-dimer tests could reduce the use of subsequent radiologic imaging to investigate patients with suspected VTE in our ED.

\section{METHODS}

Our study was a retrospective review of electronic medical records of patients presenting to the ED of an academic hospital for suspected VTE over a 2-year period. This study was part of a quality improvement initiative and exempted from obtaining informed consent by our hospital's Research Ethics Board. All patients presenting with suspected VTE in the year prior to and in the year following the introduction of a formal, standardized algorithm for investigation of VTE were included. Patient visits for suspected VTE were defined as any ED visit in which a DUS to rule out DVT, a CTPA or $\dot{V} / \dot{Q}$ scan to rule out PE, or a Ddimer test to rule out VTE was ordered.

The preintervention cohort included all ED visits from April 2, 2006, to April 1, 2007, and the postintervention cohort included all ED visits from April 2, 2007, to April 1, 2008. Exclusion criteria were any patients who had the above tests done for reasons other than suspected VTE. Although D-dimer testing was available at our university teaching hospital prior to April 1, 2007, it was used only at the discretion of the treating ED physician. On April 1, 2007, formal algorithms for the investigation of patients with suspected DVT and PE were introduced in our ED. These algorithms included a formal PTP assessment based on the Wells score (Figure 1 and Figure 2) combined with a highly sensitive latex immunoassay Ddimer (HemosIL D-dimer, Instrumentation Laboratory Company, Bedford, MA). According to the algorithm, all patients first had a clinical PTP assessment, and only those deemed low risk according to the PTP assessment were recommended to have $\mathrm{D}$-dimer testing. If the $\mathrm{D}$ dimer test was negative, then the diagnosis of VTE was considered ruled out and no further testing was recommended. Patients with a moderate or high PTP assessment were to proceed directly to appropriate imaging without D-dimer testing (see Figure 1 and Figure 2). Completion of the PTP assessment prior to the D-dimer test was mandated by a requirement that all D-dimer samples be accompanied by the PTP score sheet without which the laboratory would not run the Ddimer assay.

To identify all patients presenting to our ED with suspected VTE within the specified time frame of our study, we searched three different databases: 


\begin{tabular}{|l|c|c|c|}
\hline Clinical probability of DVT score ${ }^{1}$ & Tick if yes & Value & Score \\
\hline Inmobilized - paralysis, paresis, recent cast & & 1 & \\
\hline Cancer, active (treatment $\leq 6$ months or palliative) & & 1 & \\
\hline Pitting edema, symptomatic leg & & 1 & \\
\hline Alternative diagnosis more likety than DVT & & -2 & \\
\hline Swelling (entire leg) & & 1 & \\
\hline Tenderness (localized along deep vein or chord) & & 1 & \\
\hline Immobilized \#2 - bedridden > 3 days or surgery <1 month & & 1 & \\
\hline Veins, superficial collateral & & 1 & \\
\hline Calf swelling \& 3 em v. asymptomatio side) & & 1 & \\
\hline TOTAL SCORE & & & \\
\hline
\end{tabular}

\begin{tabular}{|c|c|c|}
\hline Score & Pretest probability & D-dimer \\
\hline$\geq 3$ & High & Do not order \\
\hline 1 or 2 & Moderate & Do not order \\
\hline$\leq 0$ & Low & Order D-dimer \\
\hline
\end{tabular}

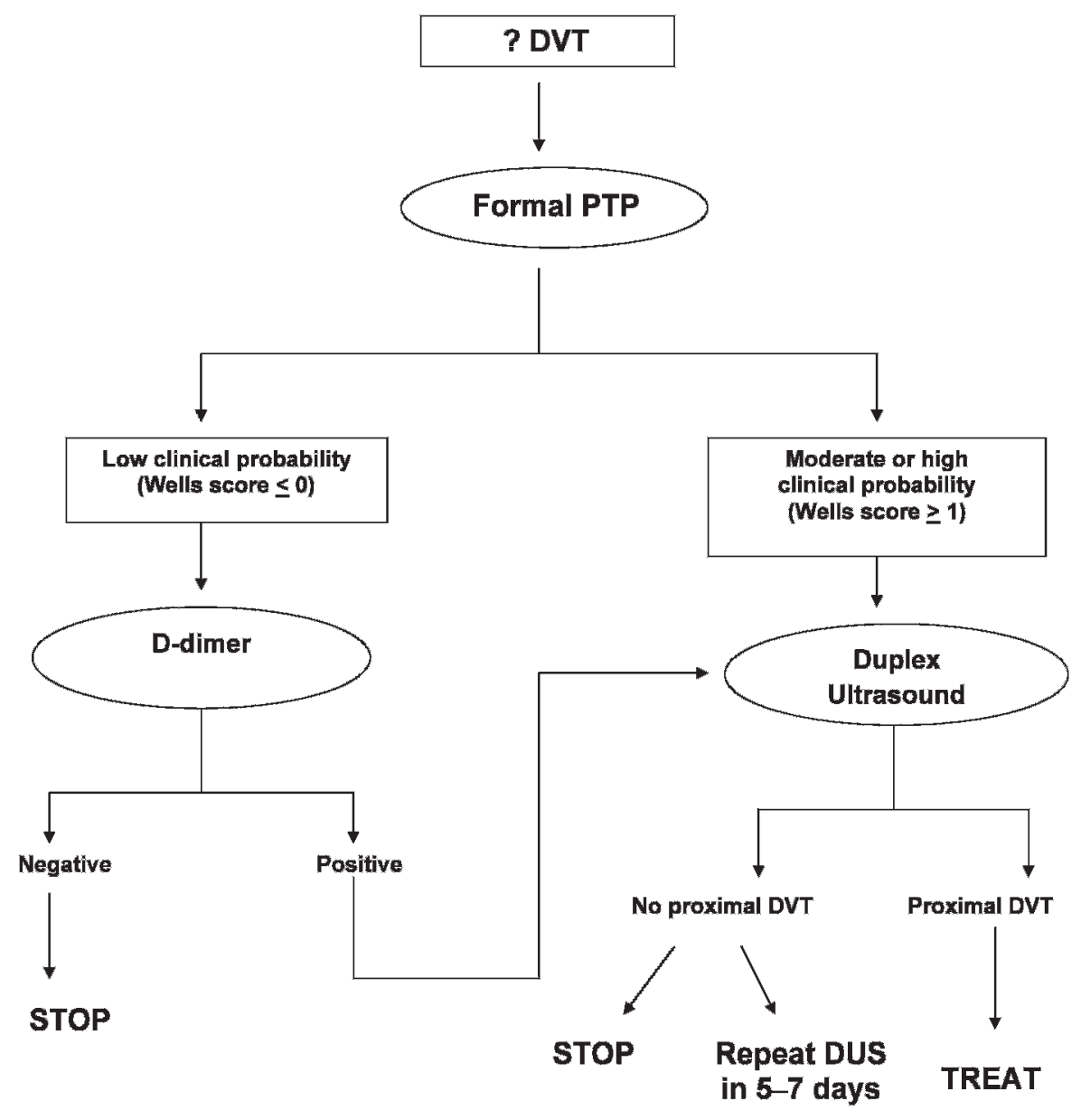

Figure 1. Deep vein thrombosis (DVT) pretest probability (PTP) assessment and algorithm. DUS = duplex ultrasonography. 


\begin{tabular}{|c|c|c|c|}
\hline Clinical probability of PE score ${ }^{1}$ & Tick if yes & Value & Sere \\
\hline $\begin{array}{l}\text { Immobilization } 23 \mathrm{~d} \text { ar surgery in } \\
\text { previsus } 1 \text { menth }\end{array}$ & & 1.5 & \\
\hline Heıaupyysis, bloody sputum & & 1 & \\
\hline Alternative diagnosis less likefly than PE & & 3 & \\
\hline DVT signs (leg swelling, lebderness) & & 3 & \\
\hline $\begin{array}{l}\text { Cancer, whive (treatrient }>6 \text { montis of } \\
\text { palliative) }\end{array}$ & & 1 & \\
\hline Previous praven DVT or PE & & 1.5 & \\
\hline Heart rale $>110$ heals per minule & & 1.5 & \\
\hline TOTAL SCORE & & & \\
\hline
\end{tabular}

\begin{tabular}{|c|c|c|}
\hline Seore & Pretest probability & D-dimer \\
\hline 26 & Hight & Do not order \\
\hline 2 to 5 & Moderate & Do not order \\
\hline 0 to 1.5 & Low & Order D-dimer \\
\hline
\end{tabular}

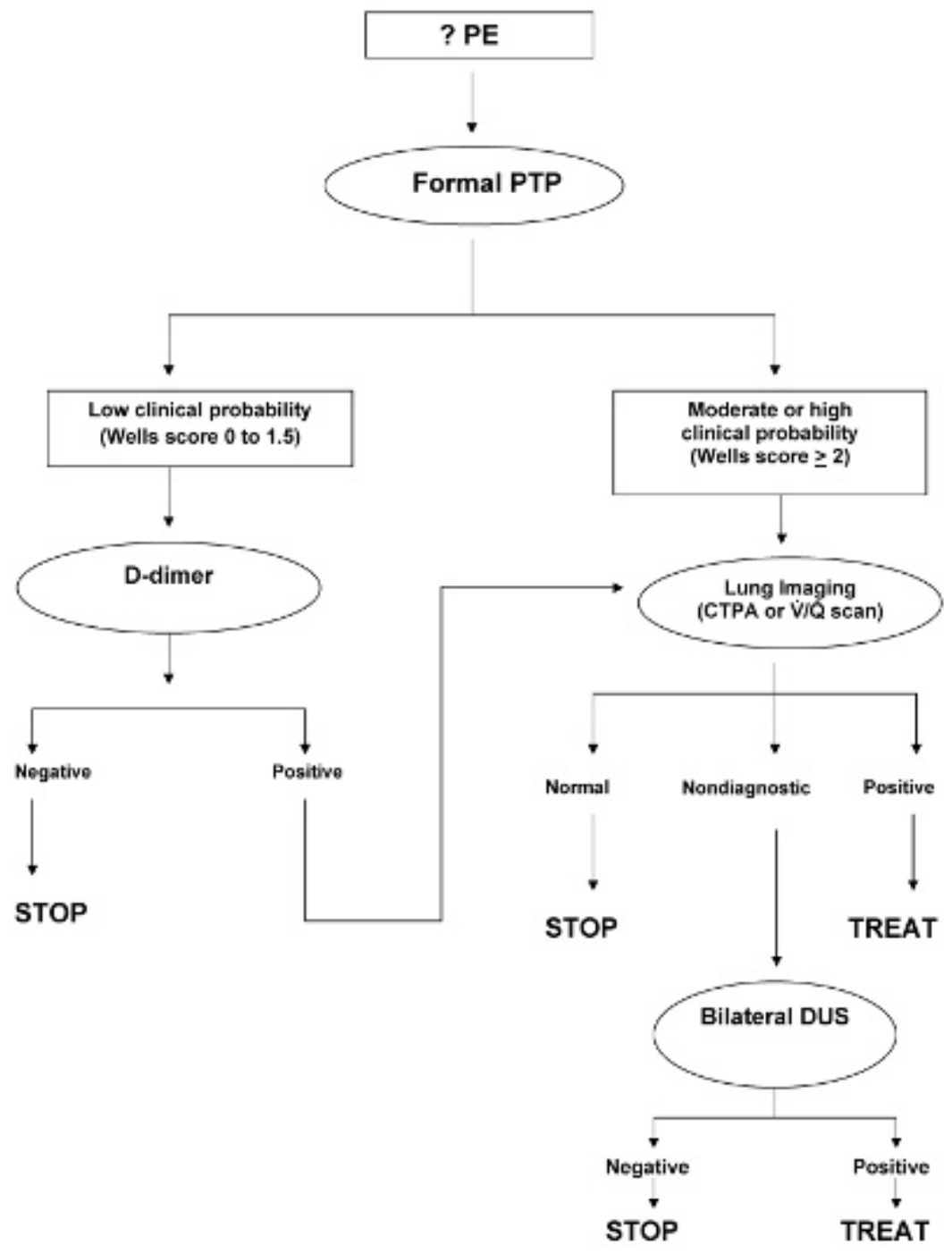

Figure 2. Pulmonary embolism (PE) pretest probability (PTP) assessment and algorithm. CTPA = computed tomographic pulmonary angiography; DUȘ = duplex ultrasonography; $\dot{\mathrm{V}} / \mathrm{Q}=$ ventilation/perfusion. 
1) administrative health records for procedure codes for DUS, CTPA, and $\dot{V} / \mathrm{Q}$ scans conducted in the ED; 2) electronic laboratory information for all D-dimers ordered in the ED; and 3) paper PTP forms from the ED that accompanied the D-dimer samples to the laboratory. We merged the lists of patients and removed any duplicate entries. The electronic patient record (EPR) was reviewed for each included patient, and the reason for ordering the $\mathrm{CT}, \dot{\mathrm{V}} / \dot{\mathrm{Q}}$, and DUS was abstracted by one author (S.I.) from the electronic requisitions, as well as other information available through the EPR. Imaging studies or D-dimers that were ordered for reasons other than to rule out VTE were excluded. For example, a chest CT scan ordered to investigate chest trauma and not to rule out $\mathrm{PE}$ and a D-dimer ordered for suspected disseminated intravascular coagulation and not to rule out VTE were excluded. Of the included patients, each imaging report was reviewed and adjudicated into three categories: positive for VTE, negative for VTE, or nondiagnostic for VTE based on the predefined criteria listed in Table 1 that were established by three of the authors (S.I., R.S., W.G.) and adjudicated by one author (S.I.). Each PTP form was reviewed by one nonblinded author (S.I.) and separated into lowprobability and non-low-probability forms. Three percent of the forms were illegible and could not be assigned into any category.

The age, sex, and proportion of patients who were admitted in each time frame studied were compared using $t$-tests and chi-square tests, respectively, depending on the variable, to ensure that there were no systematic differences from 1 year to the next in the demographics or severity of illness of the populations that were seen in our ED. The primary outcome was the total number of imaging tests for VTE performed over each of the two consecutive periods. Based on previous studies, we hypothesized that routine use of the algorithm would reduce the use of imaging tests by at least $20 \% ., 56$ Secondary outcomes were the proportion of patients with confirmed VTE (DVT or PE) after adjudication of the imaging based on the criteria listed in Table 1 . Proportions of imaging tests and confirmed DVT, PE, and total VTE in each period were compared using the chi-square test for two independent proportions, and 95\% confidence intervals (CIs) were calculated for all proportions.

\section{RESULTS}

During the prealgorithm period, there were 41,193 visits to our ED (the preintervention cohort). During

\begin{tabular}{|lll|}
\hline Table 1. Predefined adjudication criteria for confirmed venous thromboembolic disease & \multicolumn{1}{c|}{ Imaging result } \\
\hline Imaging modality & Adjudicated result & Negative \\
\hline Duplex ultrasonography & VTE not present & Positive proximal \\
& VTE present & Positive calf \\
& Nondiagnostic & Nondiagnostic \\
Computed tomographic pulmonary angiography & VTE not present & Technically adequate and no PE \\
& VTE present & Positive for segmental or more proximal PE \\
& Nondiagnostic & "Positive" for subsegmental PE \\
Ventilation/perfusion scans & Technically inadequate \\
& VTE not present & Normal \\
& Very low probability \\
& VTE present & High probability \\
& Nondiagnostic & Segmental or greater mismatch \\
& & Matched defect \\
\hline PE = pulmonary embolism; VTE = venous thromboembolism. & Low probability \\
\hline
\end{tabular}


the postalgorithm period, there were 40,680 visits (the postintervention cohort). There was no significant difference in the age ( 60 years v. 58 years), proportion of females $(61 \%$ v. $58 \%)$, or patients who were subsequently admitted ( $25 \%$ v. $26 \%)$ between the pre- and postintervention cohorts.

The electronic health record search for procedure codes for DUS, CTPA, and V/Q scans for the pre- and postintervention periods identified 3,033 codes over the 2-year period. The number of D-dimers ordered through laboratory information services during the same time was 628, and the number of PTP forms completed in the ED was 428 . After merging the lists, duplicate entries were removed, resulting in 3,499 unique ED visits (Figure 3), and for each an electronic chart review was undertaken. Of 3,499 visits, 1,676 were excluded because imaging tests were ordered for a purpose other than for ruling out VTE, and 38 were excluded because they represented multiple visits for the same VTE episode. Thus, 1,785 ED visits for suspected VTE in the pre- and postintervention cohorts were included in the analysis, of which 789 (44\%) were in the preintervention phase and 996 $(56 \%)$ were in the postintervention phase.

The proportion of ED visits associated with investigation for VTE in the postintervention cohort was higher $(2.4 \%, 95 \%$ CI 2.3-2.6) than in the preintervention cohort $(1.9 \%$; 95\% CI $1.8-2.1 ; p<0.001)$. There was no significant change in the proportion of VTE imaging tests (DUS, CTPA, and $\dot{V} / \dot{\mathrm{Q}}$ scans) ordered
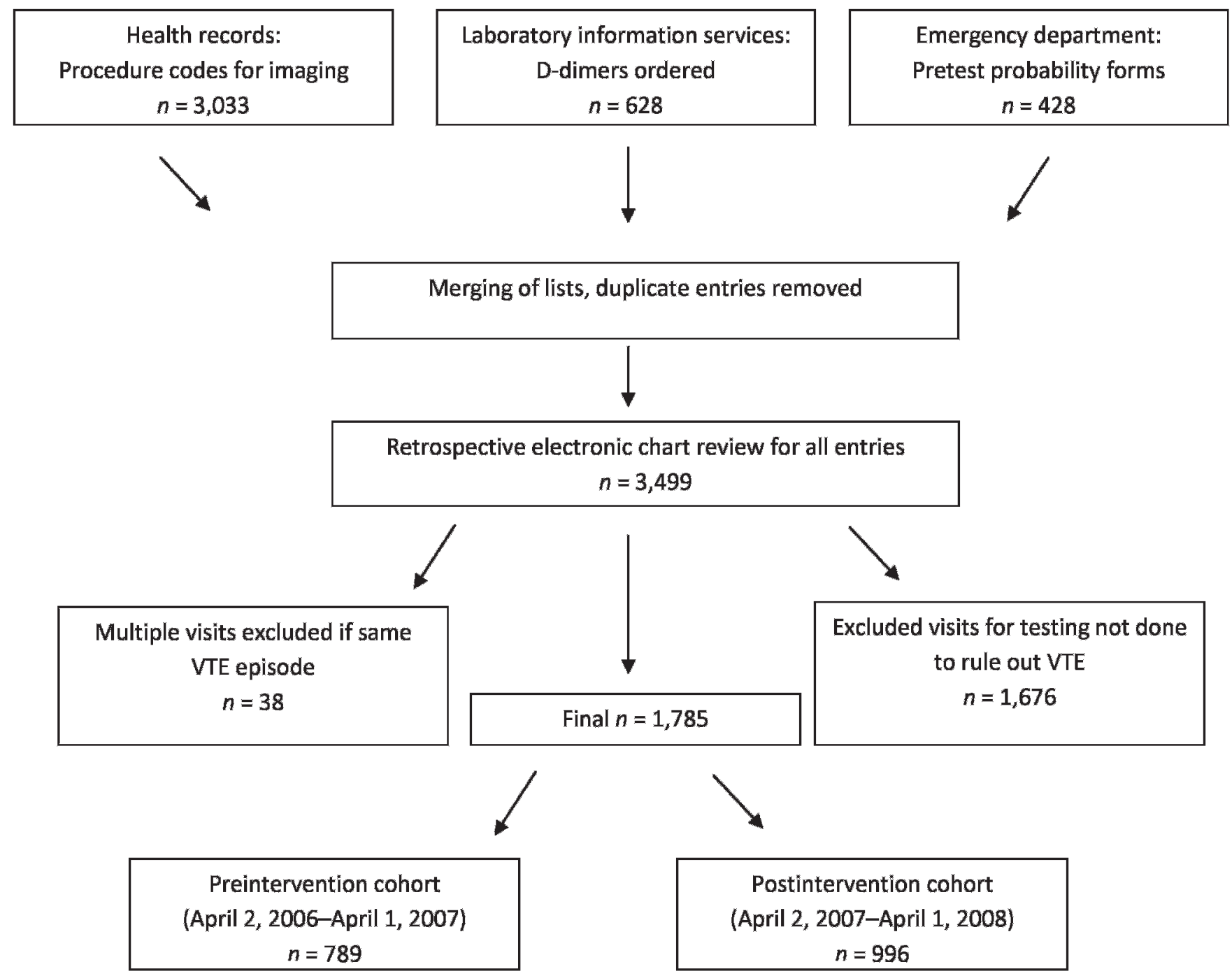

Figure 3. Methods and results. VTE = venous thromboembolism. 


\begin{tabular}{|c|c|c|}
\hline DUS, $n(\% ; 95 \%$ Cl) & Preintervention cohort & Postintervention cohort \\
\hline Total & 491 & 447 \\
\hline Negative & 424/491 (86.4; 83.0-89.1) & $376 / 447(84.1 ; 80.4-87.2)$ \\
\hline Positive (proximal) & 53/491 (10.8; 8.3-13.8) & $62 / 447(13.9 ; 11.0-17.4)$ \\
\hline Positive (calf) & 10/491 (2.0; 1.1-3.7) & $8 / 447(1.8 ; 0.9-3.5)$ \\
\hline Nondiagnostic & $4 / 491(0.81 ; 0.03-0.21)$ & $1 / 447(0.22 ; 0.04-1.25)$ \\
\hline
\end{tabular}

during all ED visits between the preintervention and postintervention cohorts, respectively (2\% v. $1.9 \%$; $p=$ 0.53 ) (Table 2, Table 3, and Table 4).

The proportion of patients with suspected VTE in whom the diagnosis was confirmed on imaging decreased overall in the postintervention cohort compared to the preintervention cohort (difference [-]: 3.8\%; 95\% CI 0.8-6.9; Table 5). This was primarily due to a reduction in confirmed $\mathrm{PE}$ postintervention $(3.8 \%$; 95\% CI 2.8-5.2) versus preintervention $(6.8 \%$; $95 \%$ CI $5.1-8.5)$ rather than DVT, which was not significantly different (Table 5).

Of the $449 \mathrm{D}$-dimers ordered in the postintervention cohort, 428 (95\%) were accompanied by a PTP form. Ninety-five percent (406 of 428) of the D-dimer requests were accompanied by forms categorized as low probability, with $2 \%$ (9 of 428) being non-low probability and 3\% (13 of 428) being illegible. Only $2 \%$ (5 of 214) of patients categorized as low PTP and negative D-dimer had imaging for VTE, and only 6\% (12 of 214) of patients with positive D-dimers for VTE did not have further imaging, suggesting that physicians were compliant with the diagnostic algorithm in more than $90 \%$ of patients. Fifty percent (214 of 428) of visits assessed as low PTP had a negative D-dimer.

\section{DISCUSSION}

The results of our study show that working with the ED and the laboratory to mandate the use of a clinical, standardized PTP assessment prior to the use of Ddimer testing is feasible, as demonstrated by the high degree of compliance with the standardized algorithm. This is in contrast to a recent report in which published guidelines were not followed stringently, with $14 \%$ of ED patients with suspected VTE and a negative D-dimer undergoing imaging and $48 \%$ with a positive D-dimer not undergoing further imaging. ${ }^{13}$ Examination of ED practice patterns at other sites has shown that fewer than $25 \%$ of physicians use a published prediction rule when investigating patients with suspected VTE. ${ }^{14}$ In a retrospective review of ED patients with suspected $\mathrm{PE}, 64 \%$ of cases had no documentation of any PTP assessment. ${ }^{15}$ Therefore, the ability to use the D-dimer assay appropriately by "mandating" a PTP assessment prior to analyzing the sample was an accomplishment.

Despite this, we were unable to demonstrate a reduction in diagnostic imaging for VTE. This finding may have been due to the following factors. First, easy access to the D-dimer assay in the context of this algorithm may have led to a lower threshold for

Table 3. CTPA in the pre- and postintervention cohorts

\begin{tabular}{lcc}
\hline CTPA, $n(\% ; 95 \% \mathrm{Cl})$ & Preintervention cohort & Postintervention cohort \\
\hline Total & 255 & 273 \\
Normal & $185 / 255(72.5 ; 66.8-77.7)$ & $205 / 273(75.1 ; 69.6-79.9)$ \\
Positive & $47 / 255(18.4 ; 14.1-23.7)$ & $35 / 273(12.8 ; 9.4-17.3)$ \\
Nondiagnostic & $23 / 255(9.0 ; 6.1-13.2)$ & $33 / 273(12.1 ; 8.7-16.5)$ \\
\hline CTPA = computed tomographic pulmonary angiography. &
\end{tabular}




\begin{tabular}{|c|c|c|}
\hline V/O scan, $n(\% ; 95 \% \mathrm{Cl})$ & Preintervention cohort & Postintervention cohort \\
\hline Total & 72 & 63 \\
\hline Normal & $46 / 72(63.9 ; 52.4-74.0)$ & $37 / 63(58.7 ; 46.4-70.0)$ \\
\hline High probability & 4/72 (5.6; 2.2-13.4) & 2/63 (3.2; 0.09-10.9) \\
\hline Nondiagnostic & $22 / 72(30.6 ; 21.1-42.0)$ & $24 / 63(38.1 ; 27.1-50.5)$ \\
\hline
\end{tabular}

considering VTE as a diagnosis and an increase in the number of patients screened for this condition. In our study, a greater proportion of $\mathrm{ED}$ visits in the postintervention period had an investigation for VTE than in the preintervention cohort. Yet the increased proportion of patients investigated for VTE did not result in an increase proportion of confirmed VTE cases. Similar results have been seen in the literature with the introduction of D-dimer in the absence of guidelines, ${ }^{9-12,16}$ in which two studies demonstrated a decreased prevalence of confirmed VTE. ${ }^{9,12}$ One study that found an increase in the number of confirmed VTE with the introduction of D-dimer found no change in recurrent VTE or in mortality, raising the question whether the additional episodes of VTE found in this manner were clinically significant. ${ }^{11}$

A second factor is that subsequent to the completion of our study, an external quality assessment survey by Quality Management Program-Laboratory Services, the Province of Ontario's external quality assessment agency for laboratories, found that the Ddimer assay we were using had a higher false positive rate in a normal, volunteer population compared to other assays in the same population. This may have impacted our study, in which $47 \%$ of the ED visits in the postintervention cohort that were assigned a low PTP had a positive D-dimer. This could have contributed to an increase in imaging without a concomitant increase in confirmed VTE. This underscores an important real-life challenge when using the D-dimer for investigation of VTE, which is establishing a valid, local cutoff value for VTE exclusion. Establishing our local cutoff value for Ddimer rather than using the manufacturer cutoff would have been important in optimizing the use of the D-dimer assay for VTE exclusion. Yet conducting such a validation study was not feasible for our laboratory and is generally not feasible outside of the research setting. It requires follow-up of patients for 3 months after being managed with a PTP plus Ddimer strategy to document the incidence of recurrent VTE.

Aside from the challenges related to establishing and validating a universal threshold, recent derivation and validation cohorts have shown that using an agedependent $\mathrm{D}$-dimer cutoff increased the proportion of patients over 50 years old in whom PE could safely be excluded. ${ }^{17}$ In our cohort with a mean age of 58 years, an age-dependent cutoff may have increased the number of low-probability patients in whom VTE could have been ruled out.

In our study, the proportion of confirmed PE decreased, whereas DVT was unchanged. This finding is seen throughout the literature. ${ }^{18,19}$ One possible explanation is that there is a relative overinvestigation of PE in patients compared to DVT because although symptoms for both are nonspecific, the case fatality associated with $\mathrm{PE}$ is higher.

Table 5. Proportion of confirmed VTE

\begin{tabular}{lcc}
\hline & Preintervention cohort & Postintervention cohort \\
\hline Confirmed VTE, $n(\% ; 95 \% \mathrm{Cl})$ & $111 / 789(14.1 ; 11.4-16.1)$ & $102 / 996(10.2 ; 8.5-12.3)$ \\
Confirmed DVT, $n(\% ; 95 \% \mathrm{Cl})$ & $57 / 789(7.2 ; 5.4-8.9)$ & $64 / 996(6.4 ; 5.1-8.1)$ \\
Confirmed PE, $n(\% ; 95 \% \mathrm{Cl})$ & $54 / 789(6.8 ; 5.1-8.5)$ & $38 / 996(3.8 ; 2.8-5.2)$ \\
\hline DVT $=$ deep vein thrombosis; PE = pulmonary embolism; VTE = venous thromboembolism. &
\end{tabular}


There are other limitations to our study. Its retrospective nature does not allow us to assess the true clinical threshold at which VTE was suspected that triggered the completion of the PTP assessment. It is also possible that the PTP form may have been inaccurately filled out simply to gain access to the Ddimer test. It is unlikely that we missed many cases of suspected VTE because we collected data from three sources and our search strategy was overinclusive (all procedures codes and $\mathrm{D}$-dimer results during that time were reviewed). Patients who were too hemodynamically unstable to have VTE investigation done would have been missed; however, the proportion of patients presenting in this manner is low, and PTP plus Ddimer testing has not been validated for use in critically ill patients.

\section{CONCLUSION}

We showed that a quality improvement initiative that mandated the use of a standardized diagnostic PTP algorithm prior to ordering D-dimer testing to rule out VTE was feasible. Despite this, use of the algorithm did not reduce imaging for VTE.

Competing interests: Rita Selby has received research support from Boehringer Ingelheim and Pfizer and honoraria from Boehringer Ingelheim, Bayer, and Sanofi Aventis. William Geerts received research support from Sanofi-Aventis, Pfizer, and Bayer Healthcare and honoraria from AstraZeneca, Calea, Eisai, Oryx Pharma, Pfizer, and Sanofi-Aventis and has worked as a consultant for AstraZeneca, Bayer Healthcare, Boehringer Ingelheim, Bristol-Myers Squibb, Covidien, Daiichi Sankyo, Eli Lilly, GlaxoSmithKline, Leo Pharma, Merck KGaA, Pfizer, Roche, and Sanofi Aventis.

\section{REFERENCES}

1. Le Gal G, Bounameaux H. Diagnosing pulmonary embolism: running after the decreasing prevalence of cases among suspected patients. 7 Thromb Haemost 2004;2:1244-6, doi:10.1111/j.1538-7836.2004.00795.x.

2. Wells PS, Anderson DR, Bormanis J, Guy F, et al. Value of assessment of protest probability of deep vein thrombosis in clinical management. Lancet 1997;350:1795-8, doi:10.1016/ S0140-6736(97)08140-3.

3. Wells PS, Ginsberg JS, Anderson DR, et al. Use of a clinical model for safe management of patients with suspected pulmonary embolism. Ann Intern Med 1998;129:997-1005, doi:10.7326/0003-4819-129-12-199812150-00002.

4. Wicki J, Perneger TV, Junod AF, et al. Assessing clinical probability of pulmonary embolism in the emergency ward: a simple score. Arch Intern Med 2001;161:92-7, doi:10.1001/ archinte.161.1.92.
5. Wells PS, Anderson DR, Rodger M, et al. Evaluation of D-dimer in the diagnosis of suspected deep vein thrombosis. $N$ Engl 7 Med 2003;349:1227-35, doi:10.1056/ NEJMoa023153.

6. Wells PS, Anderson DR, Rodger M, et al. Excluding pulmonary embolism at the bedside without diagnostic imaging: management of patients with suspected pulmonary embolism presenting to the emergency department by using a simple clinical model and D-dimer. Ann Intern Med 2001;135:98-107, doi:10.7326/0003-4819135-2-200107170-00010.

7. Ten Cate-Hoek AJ, Prins MH. Management studies using a combination of D-dimer test result and clinical probability to rule out venous thromboembolism: a systematic review. 7 Thromb Haemost 2005;3:2465-70, doi:10.1111/j.15387836.2005.01556.x.

8. Perone N, Bounameaux H, Perrier A. Comparison of four strategies for diagnosing deep vein thrombosis: a cost effectiveness analysis. Am 7 Med 2000;110:33-40, doi:10. 1016/S0002-9343(00)00598-2.

9. Lebrun E, Maitre B, Grenier-Sennelier C, et al. Effect of Ddimer testing on the diagnostic strategy of suspected pulmonary embolism: an observational study of practice patterns and costs. Eur Radiol 2000;10 Suppl 3:S433-4, doi: 10.1007/PL00014119.

10. Durieux P, Dhote R, Meyniard O, et al. D-dimer testing as the initial test for suspected pulmonary embolism. Appropriateness of prescription and physician compliance to guidelines. Thromb Res 2001;101:261-6, doi:10.1016/ S0049-3848(00)00407-2.

11. Goldstein N, Kollef MH, Ward S, et al. The impact of the introduction of a rapid D-dimer assay on the diagnostic evaluation of suspected pulmonary embolism. Arch Intern Med 2001;161:567-71, doi:10.1001/archinte.161.4.567.

12. Blais N, Morais J, Sauve N, et al. Time trends in the usefulness of pre-test prediction and D-dimer testing for the diagnosis of venous thromboembolism [abstract]. ASH Annual Meeting Abstracts, Part 1, 2006;108:943a. In: ASH; 2007.

13. Teismann NA, Cheung PT, Frazee B. Is the ordering of imaging for suspected venous thromboembolism consistent with D-dimer result? Ann Emerg Med 2009;54:442-6, doi:10.1016/j.annemergmed.2009.03.017.

14. Weiss CR, Haponik EF, Diette GB, et al. Pretest risk assessment in suspected acute pulmonary embolism. Acad Radiol 2008;15:3-14.

15. Smith C, Mensah A, Mal S, et al. Is pretest probability assessment on emergency department patients with suspected venous thromboembolism documented before SimpliRED D-dimer testing? CFEM 2008;10:519-23.

16. Girard P, Ratana S, Cosserat J, et al. Futile D-dimer testing in hospitalized patients - description, interpretation, improvements. Thromb Haemost 2008;100:1209-11.

17. Douma RA, Le Gal G, Sohne M. Potential of an ageadjusted D-dimer cut-off value to improve the exclusion of pulmonary embolism in older patients: a retrospective analysis of three large cohorts. BMF 2010;340:c1475, doi: 10 . 1136/bmj.c1475.

18. Prologo JD, Gilkeson RC, Diaz M, et al. CT pulmonary angiography: a comparative analysis of the utilization patterns in emergency department and hospitalized patients 
Ingber et al

between 1998-2003. A7R Am 7 Roentgenol 2004;182:1093-6, doi:10.2214/ajr.183.4.1831093.

19. Corwin M, Donohoo J, Partridge R, et al. Do emergency physicians use serum D-dimer effectively to determine the need for CT when evaluating patients for pulmonary embolism? Review of 5344 consecutive patients. A7R Am $\mathcal{F}$ Roentgenol 2009;192:1319-23, doi:10.2214/AJR. $\underline{08.1346 .}$ 